\title{
Flight Dynamics Simulation with Integrated Electrical System Model for Pilot-in-the-Loop Testing
}

\author{
Tuur Benoit ${ }^{1}$, Yves Lemmens ${ }^{2}$ \\ LMS, a Siemens Business, Leuven, 3001, Belgium \\ Jon Verbeke ${ }^{3}$ \\ KULAB, Oostende, 8400, Belgium \\ Rob De Roo ${ }^{4}$ \\ Vives Aerospace, Oostende, 840, Belgium
}

\begin{abstract}
Vives College University and KULAB in Belgium are undertaking an aeronautical research program about the development of a new Unmanned Aerial Vehicle (UAV). Since the UAV is completely electrically powered, the analysis of the energy management of the integrated electrical system was critical to the development of the UAV. LMS, A Siemens Business, is involved in the project to support the development of a multi-physics simulation model for electro-thermal analysis of the aircraft. This paper reports on the subsequent investigation of integrating the model of the electrical system and a multi-body model of the aircraft with landing gear for a Pilot-in-the-Loop simulation, i.e. flight simulator. The motivation is to demonstrate a unified modelling approach during the design of complex systems. The premise is that similar models based on the same modelling principals can be used during various stages of the design process. Moreover, more realistic PIL flight simulations can be attained to study the aircraft behavior and to improve pilot training. Furthermore, it enables the real-time visualisation of key variables during the simulation. The research outcome validates the feasibility and the advantages of combined models of a mechanism and electrical system for a Pilot-in-the-Loop simulation.
\end{abstract}

$\begin{array}{ll} & \\ C_{i} & =\text { aerodynamic coefficient } \\ C_{i, \alpha} & =\text { derivative of aerodynamic coefficient with respect to } \alpha \\ \alpha & =\text { angle of attack } \\ \beta & =\text { side-slip angle } \\ \delta & =\text { control surface deflection } \\ c & =\text { chord } \\ U_{\infty} & =\text { free stream velocity } \\ F_{i} & =\text { force corresponding to component-i }\end{array}$

\section{Introduction}

$\mathrm{V}$

ives College University and Kulab (KU Leuven University) in Belgium are undertaking an aeronautical research program about the development of a new Unmanned Aerial Vehicle (UAV) ${ }^{1}$. The UAV is aimed at performing scientific missions along the Belgian coast line above the North Sea. The main performance requirement of the UAV, named Litus, is a range of $160 \mathrm{~km}$ with a payload up to $15 \mathrm{~kg}$. A canard configuration with swept wings and a vertical tail at each wing tip (Figure 1) was chosen. A propulsion system of two electrical brushless DC motors had been selected in order to minimize the environmental impact during operations by avoiding gas emissions and reducing noise emissions. Test flights of the Litus are planned in the summer of 2014. The Litus has a wing span of $6 \mathrm{~m}$ and has a total mass of $65 \mathrm{~kg}$.

\footnotetext{
${ }^{1}$ Research Engineer, Aerospace Competence Centre, Interleuvenlaan 68, 3000 Leuven, Belgium.

${ }^{2}$ Sr. Project Leader RTD, Aerospace Competence Centre, Interleuvenlaan 68, 3000 Leuven, Belgium.

${ }^{3}$ Departement of Mechanical engineering, Zeedijk 101, 8400 Oostende, Belgium.

${ }^{4}$ Nieuwpoortsesteenweg 945c, 8400 Oostende, Belgium.
} 


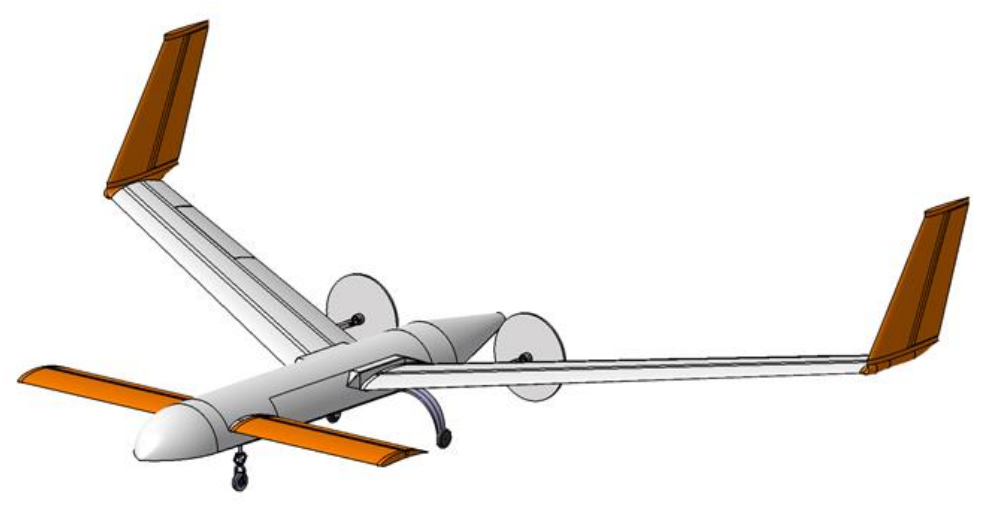

Figure 1. Vives-KULAB Litus UAV.

Since the UAV is completely electrically powered, the analysis of the energy management of the integrated electrical system is critical to the development of the UAV. LMS is involved in the project to support the development of a multi-physics simulation model for electro-thermal analysis of the aircraft. The electro-thermal system model, developed over the last two years ${ }^{2}$ was initially a stand-alone model.

This paper reports on the subsequent investigation of integrating the electrical system model with a flight dynamics model for a Pilot-in-the-Loop (PIL) simulation. Therefore, a flight dynamics model was built using the multi-body simulation software which is described in section II and 0 . In the subsequent section IV, the conversion of the model of the electrical system into a real-time simulation model is described to account for the behavior of the flight control system (FCS). Section V discussed the pilot-in-the-loop setup where the real-time multi-body and multi-physics simulation models are connected to a user input setup and visualization system. Section VI describes the simulation results. The final section draws some conclusions and presents a brief outlook for future work.

\section{Multi-body model}

The flight dynamics model was built using the multi-body simulation software to be able to include a more accurate representation of the landing gear. This will allow the simulation of a more realistic landing and ground maneuvers. The real-time solver of the multi-body simulation software LMS Virtual.Lab Motion ${ }^{3}$ was used for this. This is a dedicated C-code solver which includes many technological advancements to enable real-time solving of complex models. This solver is available for many real-time hardware platforms and uses the same input models as the standard off-line solver. It also produces result-files at the end of the simulation that can be analysed in the standard off-line environment (Figure 2).

The aircraft multi-body model (Figure 3) is composed of an airframe represented as a rigid body. The nose landing gear is composed of a simplified landing gear that is steerable. The main landing gear is an inversed Uprofile with a wheel at every leg of the ' $U$ ' and attached in the middle to the bottom of the fuselage. Clearly the flexibility of the landing gear comes from the bending of the legs. However, this is complex to model in a real-time model. Therefore, the U-profile is modelled as a rigid component but which is attached to the fuselage with a bushing which has an equivalent non-linear stiffness and damping (Figure 4).

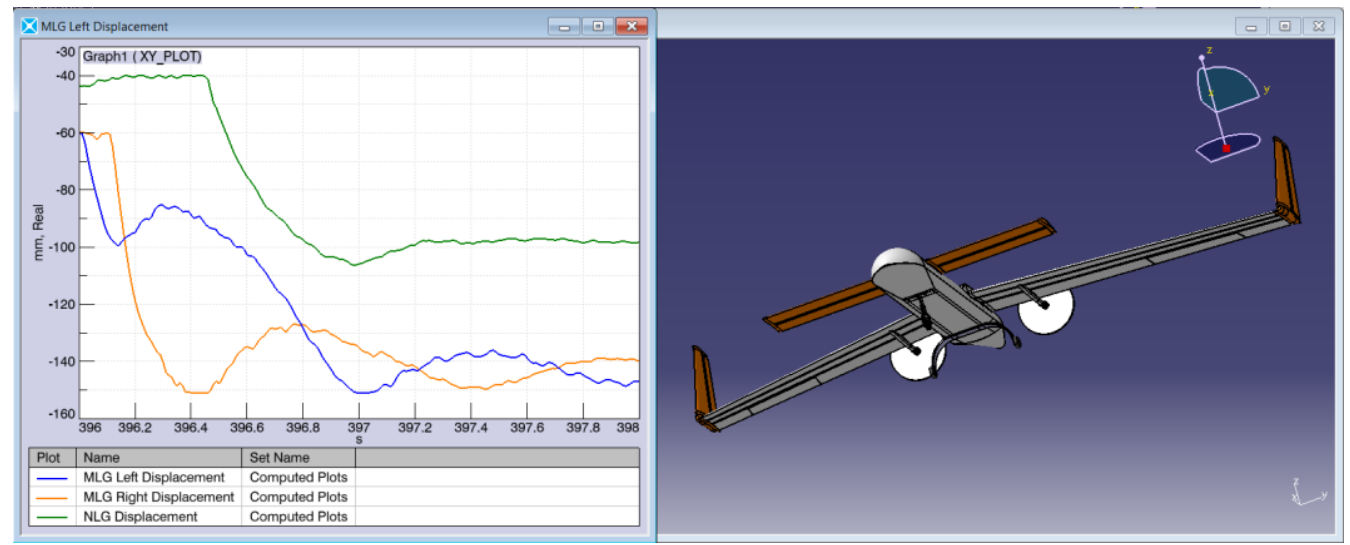

Figure 2. LMS Virtual.Lab Post-Processing interface.

American Institute of Aeronautics and Astronautics 


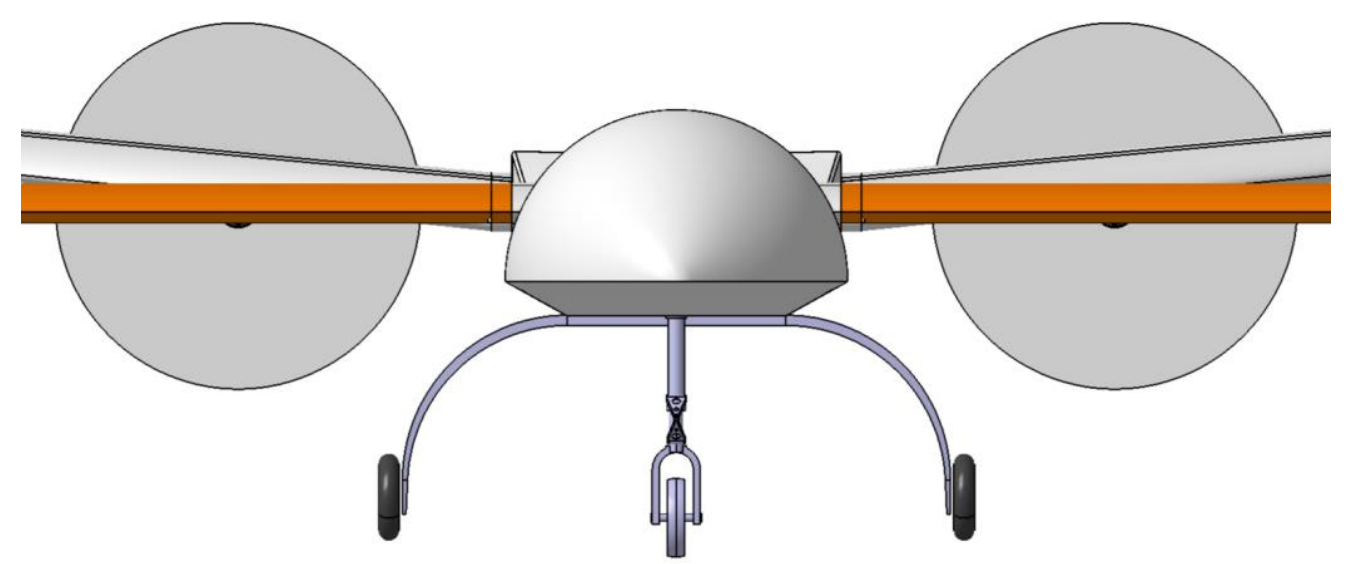

Figure 3. Multi-body model of the Vives-Litus UAV, showing the U-shaped landing gear.
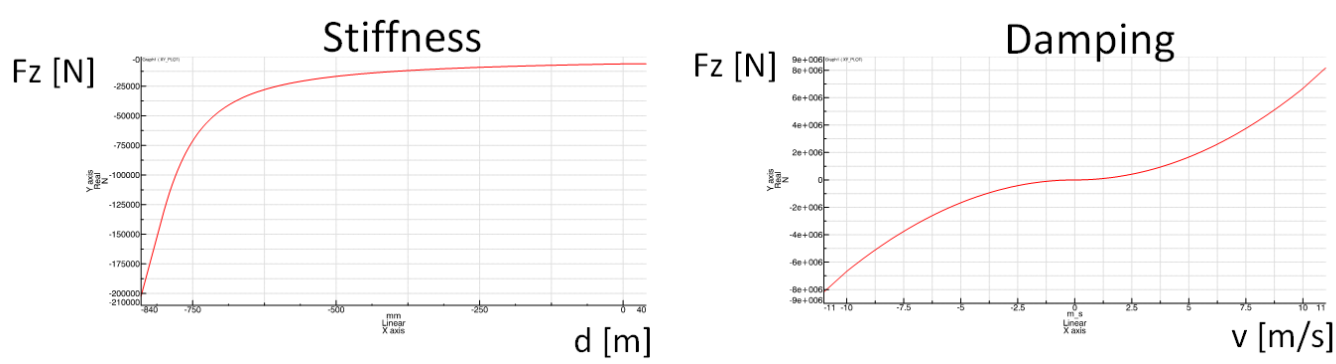

Figure 4. Nose Landing gear strut non-linear parameters.

The engines are modelled with forces elements that can also be reversed to simulate thrust reversal. The aerodynamic forces are applied at the centre of gravity and are computed is a separate module based on the attitude and speed of the aircraft. This resulted in a multi-body model with approximately 10 degree of freedom (DoFs). Even though this model has higher fidelity than used in most flight simulators, recent industrial automotive ${ }^{4}$ applications have demonstrated this approach to models with more than 150DoFs.

\section{Aerodynamics model}

The aerodynamics is computed in a separate module so that it can easily be replaced by another module for different levels of fidelity or different applications. Furthermore, the separate module can also run on a separate CPU core which parallelises the simulation and does not impact the multi-body simulation time.

The aerodynamics in this case is based on the traditional coefficient look-up method of which the main terms can be seen in equation 1 . The different terms correspond to a static coefficient, the stability derivatives and the control derivatives.

$$
C_{i}=C_{i 0}(\alpha, M, \beta)+C_{i, q}(\alpha, M, q) \cdot \frac{c q}{2 U_{\infty}}+C_{i, \delta}(\alpha, M, \delta) \cdot \delta+\cdots
$$

where:

$\alpha$ : angle of attack

$\beta$ : sideslip angle

$M$ : mach number

$\delta$ : control surface deflection

$q$ : angular rate

American Institute of Aeronautics and Astronautics 
Forces and moments at the center of gravity are consequently calculated as:

$$
\begin{array}{ll}
F_{i}=\frac{1}{2} \rho V^{2} S C_{i} & \text { for } i=L, D, Y \\
M_{i}=\frac{1}{2} \rho V^{2} S t C_{i} & \text { for } i=m, l, \mathrm{n}
\end{array}
$$

The aerodynamic coefficients have been obtained from the software CEASIOM and hence are based on the doublet lattice method. The full aerodynamic model of the UAV is described by Eqs. (3).

$$
\begin{array}{lll}
C_{L}=C_{L 0}(\alpha)+C_{L, q}(\alpha) \cdot \frac{c q}{2 U_{\infty}}+C_{L, \dot{\alpha}}(\alpha) \cdot \frac{c \dot{\alpha}}{2 U_{\infty}} & C_{l}=C_{l 0}(\alpha, \beta)+C_{l, p}(\alpha) \cdot \frac{b p}{2 U_{\infty}}+C_{l, r}(\alpha) \cdot \frac{b r}{2 U_{\infty}}+C_{l \delta_{a}}\left(\delta_{a}\right) \\
C_{D}=C_{D 0}(\alpha)+C_{D 0 \beta}(\beta) & C_{m}=C_{m 0}(\alpha)+C_{m, q} \cdot \frac{c q}{2 U_{\infty}}+C_{m, \dot{\alpha}}(\alpha) \cdot \frac{c \dot{\alpha}}{2 U_{\infty}}+C_{m \delta_{e}}\left(\delta_{e}\right) \\
C_{Y}=C_{Y 0}(\beta)+C_{Y, p}(\alpha) \cdot \frac{b p}{2 U_{\infty}} & C_{n}=C_{n 0}(\alpha, \beta)+C_{n, r}(\alpha) \cdot \frac{b r}{2 U_{\infty}}+C_{m \delta_{r}}\left(\delta_{r}\right)
\end{array}
$$

where:

$\begin{array}{lll}L: \text { lift } & S: \text { characteristic surface } & p: \text { roll rate } \\ D: \text { drag } & t: \text { characteristic length } & q: \text { pitch rate } \\ m: \text { pitching moment } & c: \text { chord } & r: \text { yaw rate } \\ Y: \text { side force } & b: \text { wingspan } & \\ l: \text { rolling moment } & V: \text { speed } & \\ n: \text { yawing moment } & & \end{array}$

\section{Electrical network}

There are 5 physical separated electric circuits in the UAV:

1. Control. This circuit controls the aerodynamic control surfaces, sets the motor rpm and adjusts the nose landing steering angle. The circuit consists of 2 receivers, 1 RRS (Redundant Receiver System), 1 Powerbox which is powered by 2 batteries, all the servos, and a motor control output signal. The receivers receive the signal from the ground transmitter. The signals are compared by the RRS and the best signal is send to the Powerbox. The Powerbox will block an output in case an error occurs such as short circuit or an over current. This provides additional redundancy. The Powerbox outputs control all servo's of the control surfaces and the nose gear. It also gives a motor setting to the speed controllers for the propulsion motors.

2. Propulsion left: This circuit supplies power to the left motor. The circuit consists of battery pack $1 \& 2$, speed controller 1 and the left motor with propeller. The speed controller gets its setting from the Powerbox. It regulates the required voltage accordingly to obtain the required rpm and thus the required thrust.

3. Propulsion right: This circuit supplies power for the right motor. This is a similar circuit as Propulsion left but with battery pack $3 \& 4$, speed controller 2 and the right motor.

4. Lights: This controls all the lights, which includes navigation, anti-collision, etc.

5. Payload: Any electrical circuits of the payload will be completely separate from the aircraft electrical circuits. Hence, it will also need separate batteries.

The electrical circuits 1, 2 and 3 are shown schematically in Figure 5. 
During the design phase of the project, simulation models of the electrical network were created with the multiphysics simulation software LMS Imagine.Lab AMESim ${ }^{5}$. The electrical system that will be used during the test flights was completely modelled. This included models of the energetic behavior of all main electrical components:

- 2 receivers

- $\quad$ RRS

- Powerbox

- 13 servos

- 2 motors

- 2 ESCs

- 2 propellers

- 4 battery sets

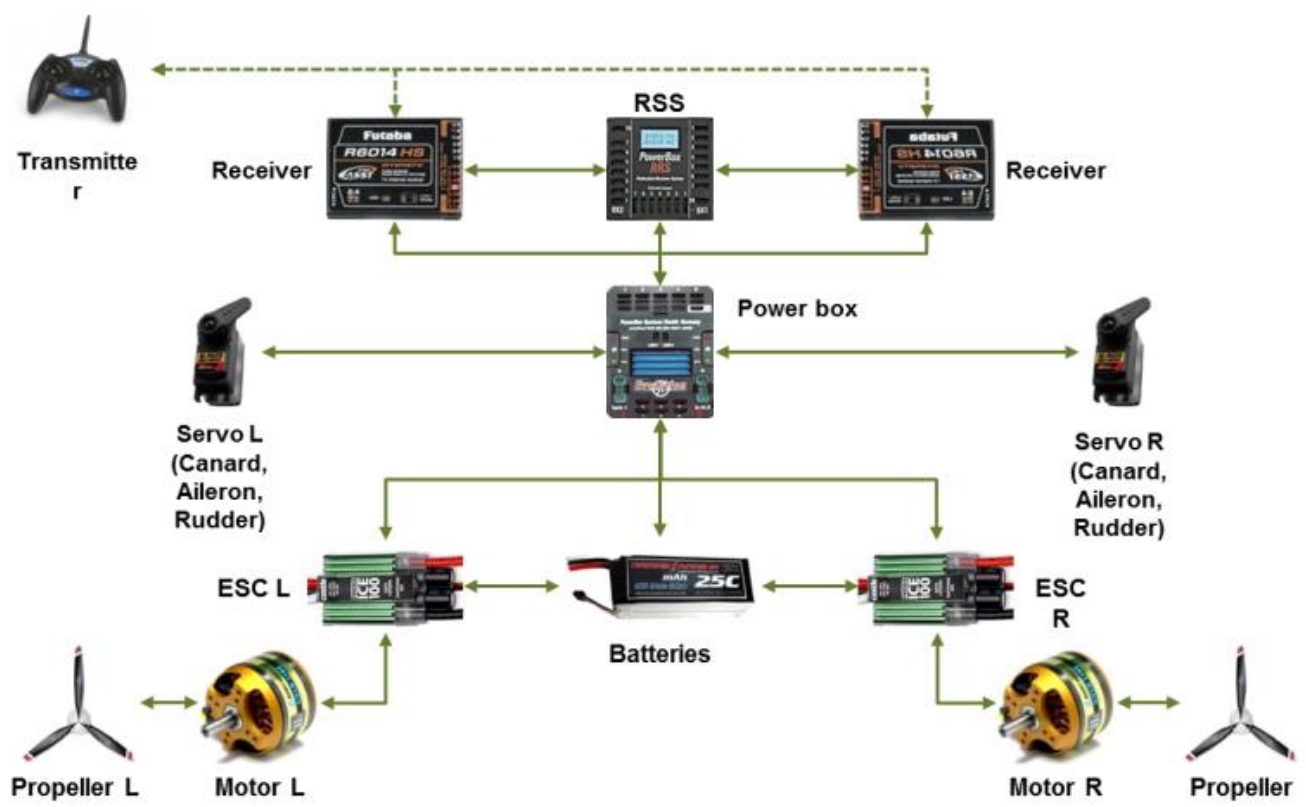

Figure 5. Vivis Litus electrical network architecture 


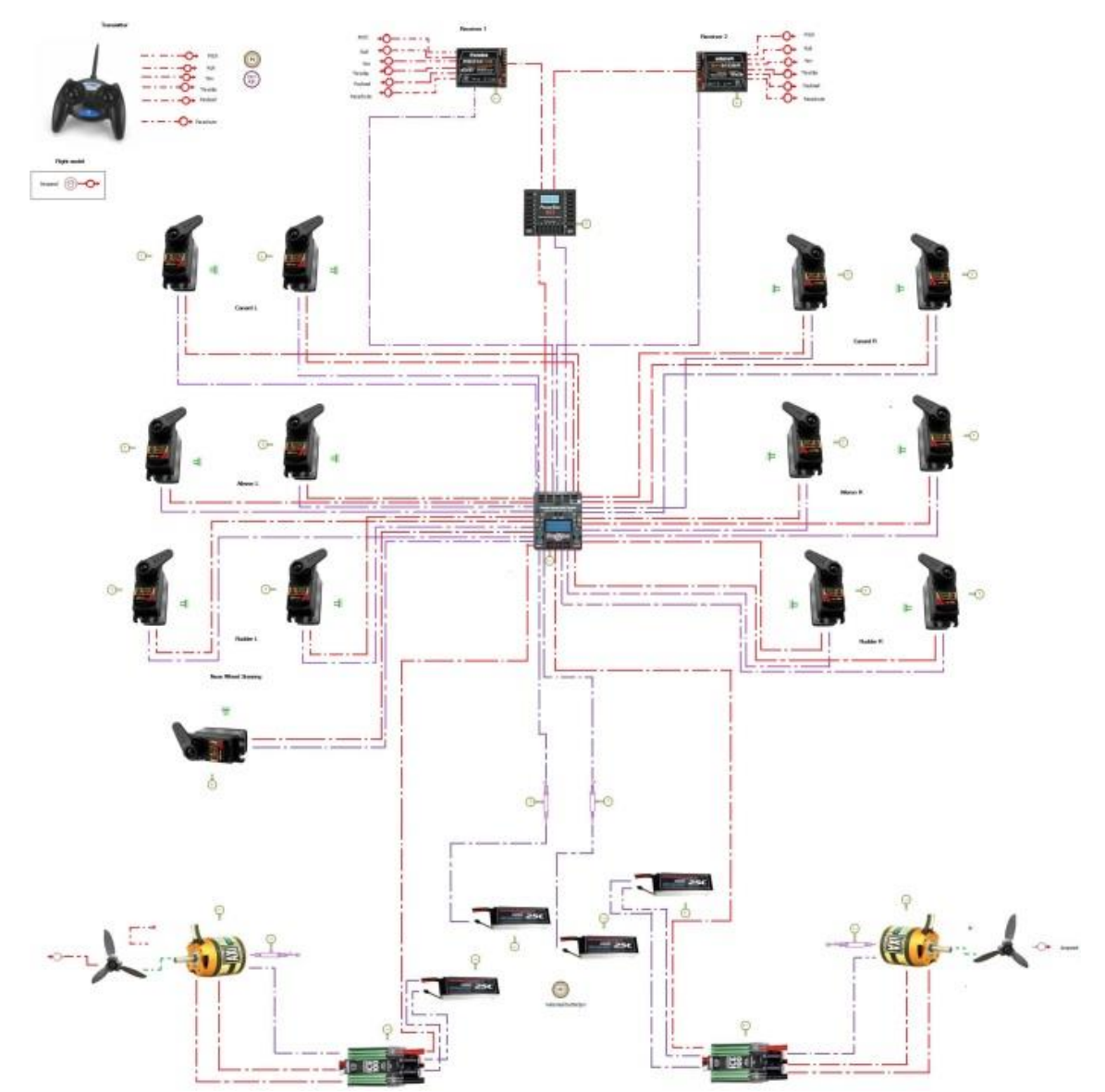

Figure 6. Simulation model of electrical network in LMS Imagine.Lab AMESim [5].

Also the input control signals were modelled and are used to control the behavior of the electrical system. The objective of the electrical system model was to support the sizing of the components and batteries to achieve the required performance. Therefore, the modelling of the components focused on their energetic and not on their dynamic behavior. The energetic behavior of all components was validated on test benches. This included a test bench of the motor with propeller for which also the propeller torque and thrust were measured, together with the power consumption of the motor and its electronic speed control (ESC). As a result, a validated electrical system model was obtained. The top-level view of the simulation model is shown in Figure 6.

Subsequently, the reuse of this model was investigated for the PIL simulations. However, not all simulation models can be run in real-time. First of all, the total duration of the simulation needs to be less than the time that is simulated but more importantly, the duration of every time step of the simulation should be faster than the simulated time. This means that very fast transient effects are difficult to simulate in real-time and hence the model should be adopted accordingly. This is in particular an issue when dealing with models of electrical networks. Finally, the component elements that are used in the simulation model should have no internal-states as this creates additional overhead and slows down the computation. This can be avoided by selecting alternative simulation models of a component.

In the case of the electrical network model of the Litus, all component elements used already are in form of a quasi-static description, except for the batteries. This means that the models did not include fast transient effects as this was not required for the energy management and thermal management analyses for which it was used. On the contrary, the models of the servo motors were extended to capture better their mechanical dynamic behavior. Additionally, the models of batteries and thermal wire models for the main motors were changed to eliminate implicit-states (Figure 7). Additional simulations that compare the RT and off-line models confirmed that they result in the same behavior of the electrical network except for the servo properties. 


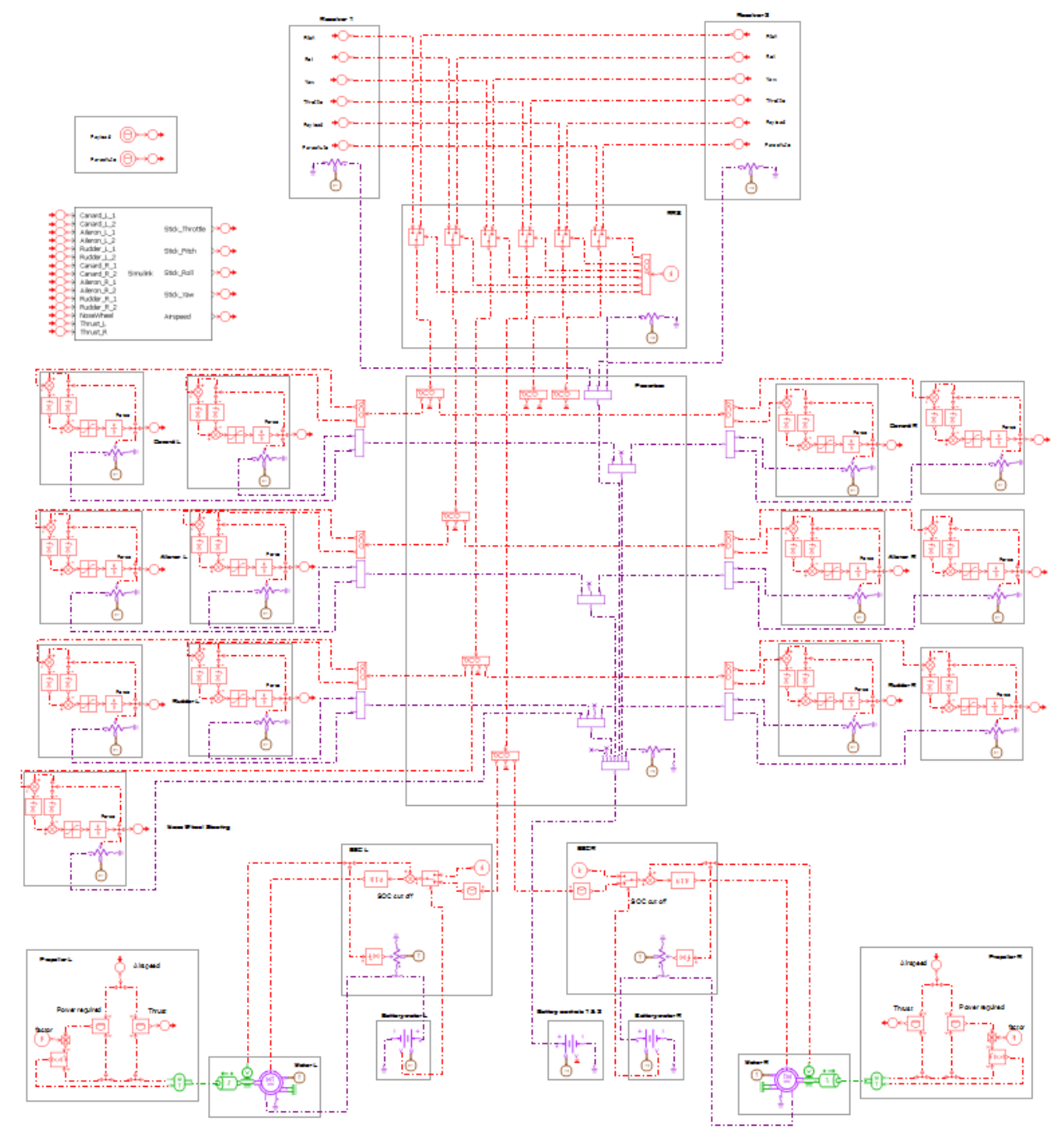

Figure 7. Real-time simulation model of electrical network.

\section{Pilot-in-the-Loop setup}

The demonstrator setup is shown in Figure 8. There are two computer systems. There is the client system with which the user interacts. It is connected to the input devices which are a stick, throttle lever and a pair of pedals. The interface with the input devices is based on the Active $\mathrm{X}^{\complement}$ MS Windows driver.

Also on the client system is the visualisation software. In this case, there are two types of visualisation. First there is the open-source software FlightGear ${ }^{6}$ which shows the cockpit view. Of course, the flight dynamics algorithm was removed from FlightGear and this information comes now from the multi-body software. Secondly, an additional bespoke software was used to take advantage of the high-fidelity aircraft model. This software shows in real-time key variables or states of the aircraft. For example in the case of this demonstrator, the compression stroke of the struts in nose landing gear can be shown. 


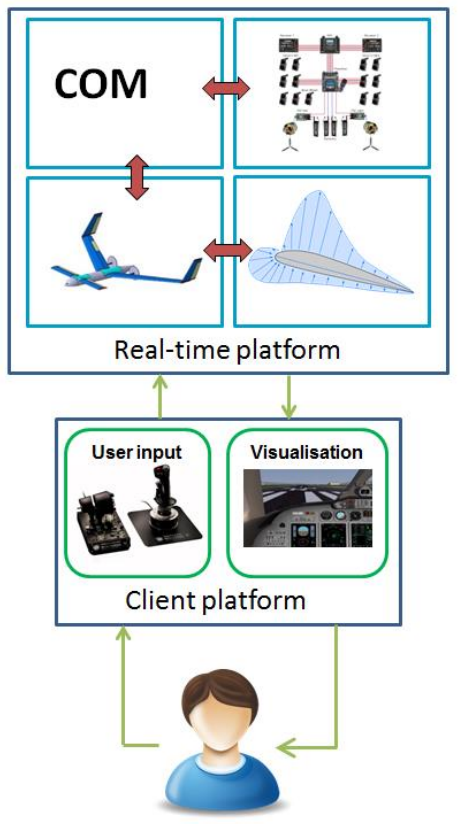

Figure 8. Proposed Simulation architecture.

The second computer system is the platform for the real-time simulation. It is a combined simulation architecture based on 4 parallel processes (Figure 8). The first process is the communication module that it controls the time scheduling of the coupled real-time simulation. The second process is the simulation of the electrical system based on the existing electric model. The model inputs are the flight control inputs from the user and throttle settings, while the outputs are the angles of the flight control surfaces and the thrust of the propellers. These model outputs are used as input to the multi-body simulation software which is the third process. The multi-body simulation output is the attitude of the aircraft that is then sent to the visualization. The multi-body simulation code is also coupled to an aerodynamic module that computes the aerodynamic force in the center of gravity of the aircraft. This aerodynamic module is the fourth process.

The communication between the two platforms is based on the standard UDP network protocol. Table 1 shows in the left column the input parameters from the user to the real-time solver and the right column shows the output that is used by the two visualisation software.

Table 1. Overview multi-body dynamics solver, inputs and outputs.

\begin{tabular}{|l|l|}
\hline Solver input & Solver output \\
\hline Aileron / elevator / rudder & Aircraft CoG position / orientation \\
Steering angle & Aircraft CoG velocity \\
Thrust & Compression stroke of landing gear \\
Flaps & \\
\hline
\end{tabular}

\section{Simulation results}

A mission profile, as shown in Figure 9, was flown with the flight simulation that was described before. It is composed of 5 phases. First is the take-off phase duration which the throttle is set to maximum (100\%). This phase takes about 10s. Subsequently, there is the climb phase where the throttle is set to $90 \%$ and the elevators setting are increased. The climb phase is performed for about 100s. The next phase is the cruise phase where the thrust is set to $75 \%$ and the elevator settings are reduced. This is maintained for about 400s. Finally, there are the descent and landing phases where the thrust of the engines is further reduced.

The thrust recorded from motor 1 during the take-off, climb and cruise phases is shown in Figure 10. The corresponding state-of-discharge of the battery pack is shown in Figure 11. During take-off and climb, the thrust setting is higher than in cruise phase. In addition, the power consumption is also higher, resulting in a faster discharge of the batteries. 


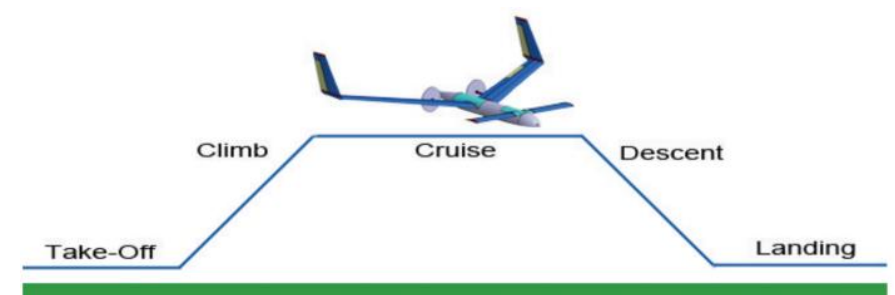

Figure 9. Mission profile flown with flight simulator.

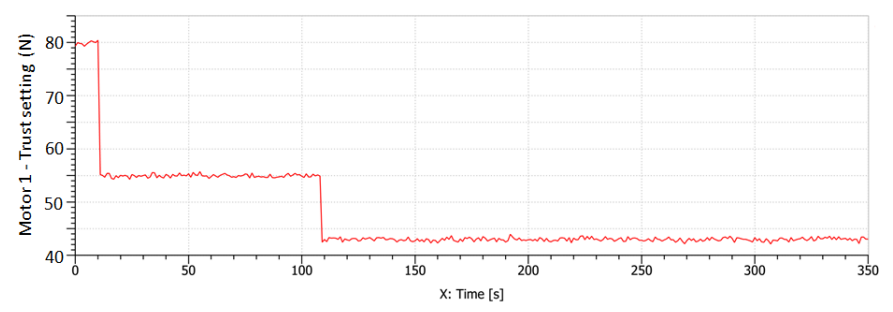

Figure 10. Recorded data for thrust of motor 1 (Take-off \& cruise).

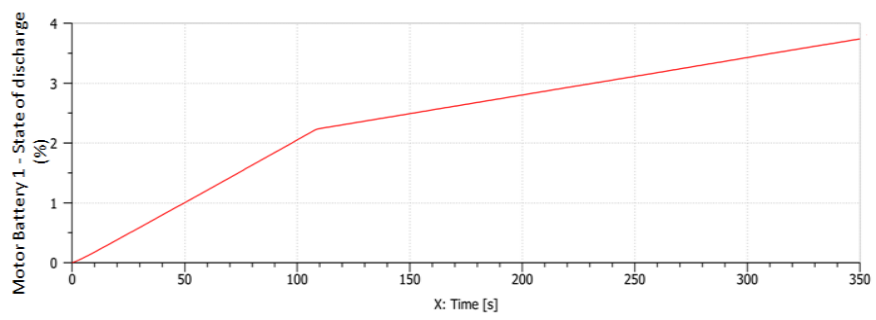

Figure 11. Recorded data for state of charge of battery 3 and 4.

Results regarding the flight dynamics are attained from the multi-body model of the aircraft. During the last four seconds of the simulation, the aircraft performs a landing on the runway. Figure 12 shows the vertical displacement of the wheels. The landing is quite asymmetric since the initial touch down is on the left main landing gear only. Slightly afterwards, also the right main landing gear touches the runway. Finally after rotation, the nose landing gear reaches the ground.

Figure 13 shows that initially the left main landing gear is only compressed slightly, resulting in low spring forces. The right main landing gear is compressed more, since it has to conteract the rotational inertia effects of the aircraft resulting from the asymmetric landing.

After touch down, while driving the runway, the aircraft oscillates heavily in lateral direction. This phenomenon is caused by the highly non-linear characteristics of the damping of the landing gear (Figure 14). High damping forces are present during the first contact with the ground, but only very low damping forces during the ground run. Our experience with the PIL-simulator has shown that pilots can have trouble steering an aircraft, with such an undamped landing gear shock absorber, on the ground. During high speed turns the aircraft tends to stick in its turn, caused by the non-linear damping, while after unsticking high lateral oscillations make the control harder. 


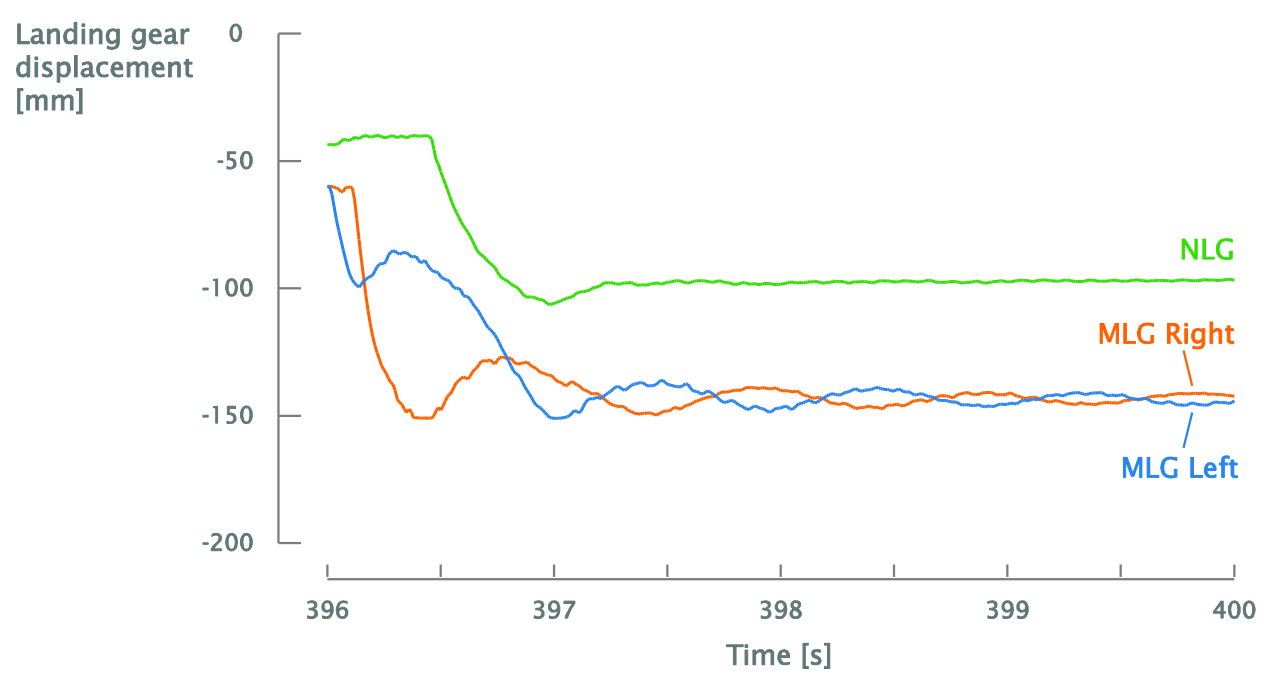

Figure 12. Displacement of the main (MLG) and nose landing gear (NLG).

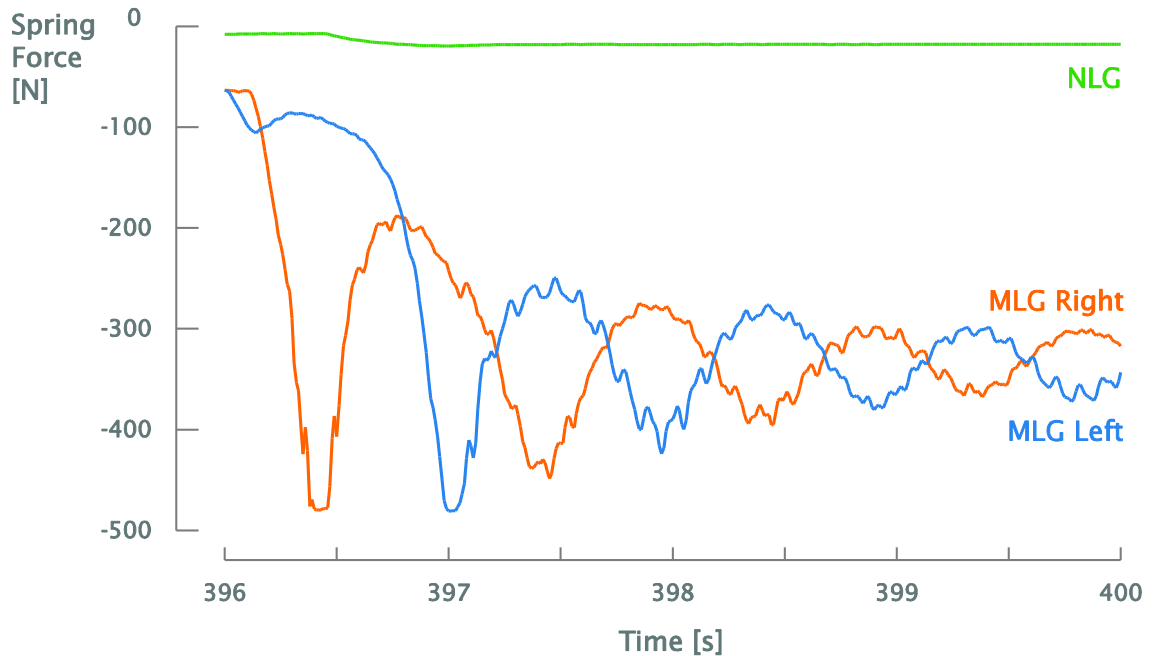

Figure 13. Spring force of the main (MLG) and nose landing gear (NLG).

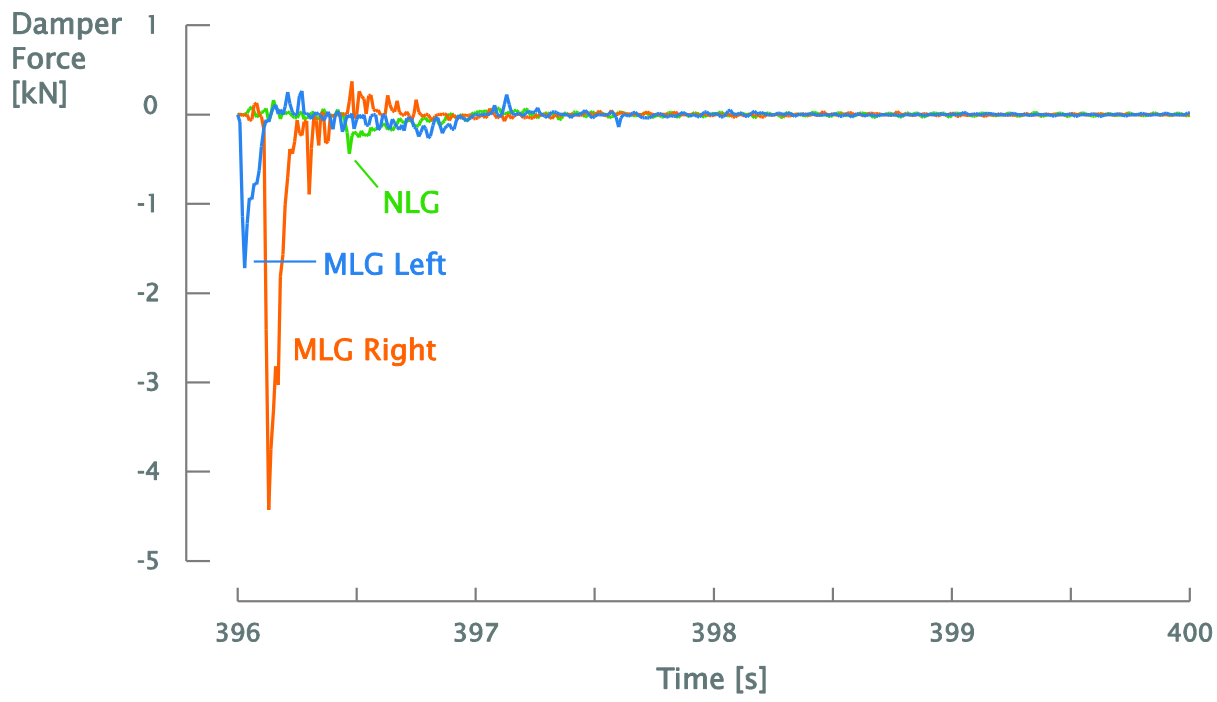

Figure 14. Damping force of the main (MLG) and nose landing gear (NLG).

10

American Institute of Aeronautics and Astronautics 


\section{Conclusion}

This paper reported on recent advances in multi-body and multi-physics simulation software that enables the real-time simulation of detailed aircraft models. The motivation is to support a unified modelling approach during the design of complex systems. The premise is that similar models based on the same modelling principals can be used during various stages of the design process. Moreover, more realistic PIL flight simulations can be performed to studies that aircraft behavior and an improved training and key variables can be visualized in real-time during the simulation.

The paper presented a case study of a UAV. The simulation model was composed of a multi-body model that included a landing gear and multi-physics model of the electrical propulsion and flight control system. Both models were coupled together for demonstrator PIL setup. The simulatin results demonstrated the feasibility and advantages of the setup.

In the future, an investigation is anticipated to couple the same model of the electrical network and flight dynamics module to an autopilot hardware platform in order to tune and validate the autopilot performance. This would demonstrator a Hardware-in-Loop (HIL) application.

\section{Acknowledgments}

We kindly acknowledge IWT Vlaanderen for their support of the ongoing research project "Model Driven Physical Systems Operation - MODRIO", which is part of the ITEA2 project 11004 "MODRIO" (in turn, supported by the European Commission), and of the IWT Baekeland project 130838.

\section{References}

${ }^{1}$ Becuwe, I., De Roo R., Verbeke, J., “PWO UAV Project - Final Report”, November 2012.

${ }^{2}$ Lemmens, Y., Verbeke, J., De Roo, R., Becuwe, I., Olbrechts T., "Simulation and validation of energy and thermal management of an electrically-powered UAV", 2012 SAE Power Systems Conference, Phoenix, Arizona, USA, October 30 - November 1, 2012.2.

${ }^{3}$ Siemens PLM, LMS Virtual.Lab Rev12 Manual, 2013

${ }^{4}$ Prescott, W., Heirman, G., Furman, M., De Cuyper, J., Lippeck, A., Brauner, H., "Using High-Fidelity Multibody Vehicle Models in Real-Time Simulations", SAE 2012 World Congress \& Exhibition, Detroit, Michigan, United States, April 24, 2012.

${ }^{5}$ Siemens PLM, LMS Imagine.Lab AMESim Rev13 Manual, 2013.

${ }^{6}$ Flightgear website "http://www.flightgear.org/" retrieved 01.04.2014 